\title{
Comparação de Algoritmos para Detecção de Nadadores Visando Automação de um Veículo Elétrico
}

\author{
Lucas Correa de Assis \\ Engenharia de Controle e Automação, Escola Politécnica, \\ Pontifícia Universidade Católica do Paraná - PUCPR \\ Curitiba, Brasil \\ assis.correa@pucpr.edu.br
}

\author{
Roberto Zanetti Freire \\ Programa de Pós-Graduação em Engenharia \\ de Produção e Sistemas - PPGEPS, Escola Politécnica, \\ Pontifícia Universidade Católica do Paraná - PUCPR \\ Curitiba, Brasil \\ roberto.freire@pucpr.br
}

\begin{abstract}
Na análise biomecânica de nadadores, o uso de câmeras vem contribuindo para a melhora de rendimento esportivo. Porém grande parte dos vídeos são gravados de forma manual. Este trabalho tem como objetivo realizar uma análise comparativa entre os algoritmos de aprendizado profundo, YOLO v4 e YOLO v4 Tiny, para detecção de nadadores, visando o projeto de um veículo capaz de seguir, de forma autônoma, os atletas ao longo da piscina. $O$ treinamento dos algoritmos foi realizado com a base de dados Video Diver Dataset (VDD-C), que contém imagens anotadas de mergulhadores em piscinas e na costa de Barbados no Caribe. Para comparação entre os algoritmos, levou-se em consideração qualidade e tempo de detecção, baseando-se nas métricas de Average Intersection Over Union ( $I o U)$, Recall, Average Precision (AP) e tempo de latência. O algoritmo $\mathrm{YOLO} v \mathrm{v}$, apresentou superioridade na qualidade de detecção, atingindo $71,38 \%$ de Average IoU. Porém a YOLO v4 Tiny, apresentou um tempo de latência 6,93 vezes menor, mostrando ser uma opção para sistemas embarcados e aplicações em tempo real.
\end{abstract}

Index Terms-Aprendizado Profundo, Análise Biomecânica de Nadadores, Detecção de objetos, YOLO.

\section{INTRODUÇÃO}

A análise biomecânica, se tornou um instrumento de grande importância para ajudar a melhorar o desempenho de nadadores. Em [1], enfatiza-se a importância de registrá-los por meio de vídeo, principalmente no que se refere à alta demanda em termos de precisão associada a este tipo de análise [2]. Em sua maioria, os vídeos são gravados manualmente, acompanhando o nadador ao longo da piscina, ou por uma estrutura fixa de câmeras, conforme apresentado em [3] para estimar a pose humana em vídeos de nadadores.

Para a automação do processo de aquisição de imagens do nadador, é necessário desenvolver um sistema de visão computacional capaz de gerar referências de velocidade e posição para um veículo elétrico, que acompanhará o nadador ao longo da piscina.

Nesse contexto, este artigo considera o uso de técnicas de visão computacional para detecção de nadadores, como apresentado em [4], na abordagem multi-related-targets, e por [5] na análise de modelos de aprendizado profundo para detecção de mergulhadores. Assim, o presente trabalho tem como objetivo avaliar o algoritmo de aprendizado profundo YOLO (You Only Look Once) [6], a partir da análise comparativa entre as versões YOLOV4 e YOLOV4 Tiny [7] para a detecção de nadadores.

A próxima seção deste artigo apresentam os trabalhos relacionados que serviram de inspiração para o desenvolvimento deste estudo. Na sequência, a seção III apresenta a base de dados, as técnicas adotadas e os critérios de avaliação de desempenho adotados neste trabalho. A seção IV apresenta os experimentos realizados e a seção $\mathrm{V}$ os resultados obtidos. Por fim, a seção VI traz as conclusões do presente estudo.

\section{TRABAlhos Relacionados}

Os trabalhos apresentados nesta seção serviram de inspiração no desenvolvimento deste estudo.

No estudo apresentado em [4], foi desenvolvido o método multi-related-targets para tracking de nadadores. O método considera o nadador como um conjunto de subtargets que avançam na mesma velocidade, possibilitando a estimativa da localização de subtargets parcialmente ou totalmente oclusos. $\mathrm{O}$ método apresentou acurácia superior à abordagem clássica de tracking apenas da cabeça do nadador.

Já no trabalho apresentado em [1], foi investigado o método contrario para detecção de nadadores, que consiste na modelagem do ruído aleatório da água e detecção do movimento estruturado do nadador. Para isso, apresentaram a técnica DLT (Direct Linear Transform) para calibrar o vídeo, onde foi extraído a raia do nadador. Na sequência, foi aplicada a subtração de frames para detecção do movimento, decompondo o frame em um grid e classificando cada célula do grid como nadador ou ruído. Os testes conduzidos mostraram bons resultados para localização e tracking de 
nadadores.

$\mathrm{Na}$ mesma linha dos trabalhos anteriores, em [5] foram avaliados modelos de aprendizado profundo para detecção de mergulhadores, produzindo um dataset com mais de 105.000 imagens anotadas de mergulhadores, que foi utilizado para treinar redes neurais como Single Shot MultiBox Detector (SSD), Mobilenet, Faster R-CNN e YOLO. Tomando como base os resultados desses testes, os autores reportaram resultados relevantes com o uso da SSD e da YOLO v4 Tiny para aplicações em tempo real.

No estudo discutido em [8], foi introduzido o modelo YOLO LITE desenvolvido para ser executado em dispositivos sem a presença de uma Graphical Processing Unit (GPU). O modelo foi treinado com a base de dados PASCAL VOC [9] e posteriormente com a base de dados COCO [10], atingindo valores de mean Average Precision (mAP) de 33,81\% e $12,26 \%$, respectivamente. A YOLO LITE atingiu a marca de 21 Frames per Second (FPS) em computadores sem GPU e 10 FPS quando implementado em um site com apenas 7 camadas. Esta taxa de FPS é 3,8 vezes mais rápida, quando comparado ao modelo mais rápido SSD Mobilenet $v 1$.

Seguindo a mesma proposta do trabalho anterior. $\mathrm{O}$ trabalho apresentado em [11], propõe um novo framework chamado Fast YOLO, que acelera a YOLO v2 [12] para ser capaz de realizar detecção de objetos em sistemas embarcados para aplicações em tempo real. Neste caso, produziram uma arquitetura otimizada que possui 2,8 vezes menos parâmetros e uma queda de apenas $2 \%$ nos valores de Intersection Over Union $(I o U)$. Resultados experimentais mostram que a Fast YOLO é capaz de aumentar a velocidade de detecção em média 3,3 vezes, se comparado a YOLO v2 originalmente. Possibilitando a detecção de vídeos com taxa média de 18 FPS em um sistema embarcado na Nvidia Jetson TX1.

\section{MATERiAis E MÉtodos}

Esta seção descreve a coleta e tratamento dos dados, os algoritmos de aprendizado profundo e as métricas de avaliação dos modelos.

\section{A. Base de Dados}

A base de dados utilizada neste artigo é denominada Video Diver Dataset (VDD-C) [5]. que contêm 100.000 imagens anotadas de mergulhadores em piscinas e na costa de Barbados no Caribe, ambas embaixo d'água. Como o foco deste artigo é detectar e classificar nadadores, utilizou-se apenas as imagens coletadas em piscinas (Fig. 1), totalizando 5.620 imagens.

As imagens foram divididas em três conjuntos, o conjunto de treinamento que representa $71,91 \%$, o conjunto de teste que representa $14,86 \%$, e o conjunto de validação que representa $13,23 \%$. Conforme apresenta a Tabela I.
Fig. 1: Representação das imagens em piscinas do dataset $V D D-C$.

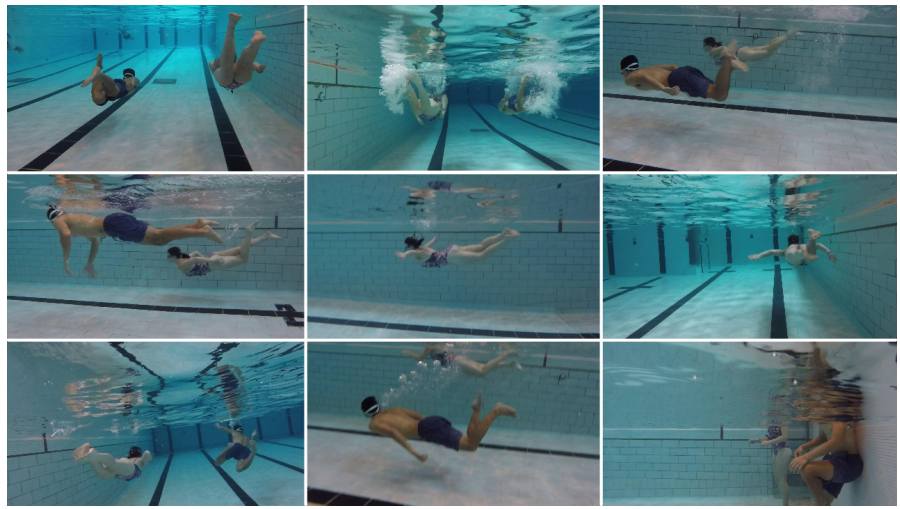

Tabela I: Distribuição dos dados

\begin{tabular}{ccc}
\hline Categoria & Número de Imagens & Percentual \\
\hline Treino & 4.041 & $71,91 \%$ \\
Teste & 835 & $14,86 \%$ \\
Validação & 744 & $13,23 \%$ \\
\hline
\end{tabular}

\section{B. YOLO (You Only Look Once)}

Redmon et al. (2016) introduziram uma nova abordagem para a detecção de objetos com o algoritmo YOLO [13], reformulando a solução para uma regressão única, diretamente dos pixels da imagem, para a localização e classificação dos objetos. Por apresentar apenas uma rede neural convolucional para toda imagem, a $Y O L O$ é considerada rápida e permite detecções em tempo real.

A YOLOV4 é composta pela estrutura CSPDarknet53 [14], o módulo de Spatial Pyramid Pooling (SPP) [15], o ramo de Path Aggregation Network (PAN) [16], e a parte superior que é baseada na YOLOV3 [6], que utiliza a arquitetura Darknet53 com 53 camadas convolucionais para classificação e outras 53 camadas de detecção, totalizando 106 camadas de profundidade.

Para a detecção, a imagem é dividida em um grid, onde cada bloco possui três regiões de detecção (Boundig Boxes). Cada uma delas possui suas coordenadas (Box Co-ordinates), a probabilidade de possuir um objeto (Objectness Score) e as probabilidades das classes (Classes Score), conforme Fig. 2.

Onde $t_{x}, t_{y}, t_{w}$ e $t_{h}$ representam a coordenada $x$ do centro, a coordenada $y$ do centro, a largura e altura relativa da região de detecção em relação a coordenada superior esquerda do bloco do grid, respectivamente. $p_{o}$ representa a probabilidade da região possuir um objeto, seguido de $p_{1}, p_{2} \ldots p_{c}$ que representam as probabilidades de cada classe para aquela região, tendo $c$ como número de classes. 
Fig. 2: Representação do grid na imagem e dos dados contidos em uma região de detecção (adaptado de [17]).

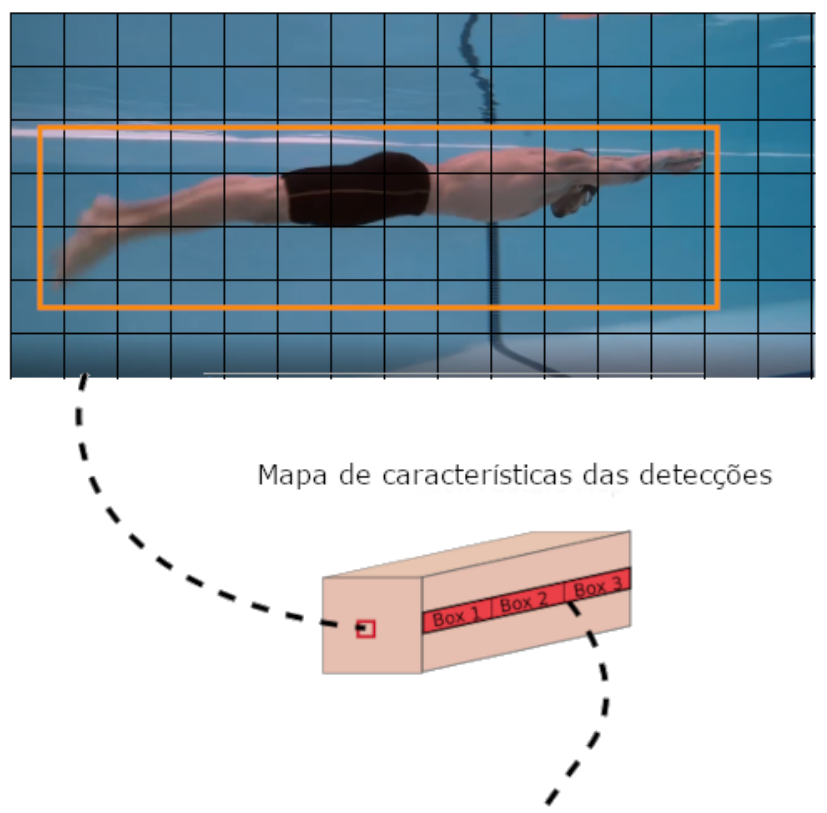

Atributos de uma deteç̧ão

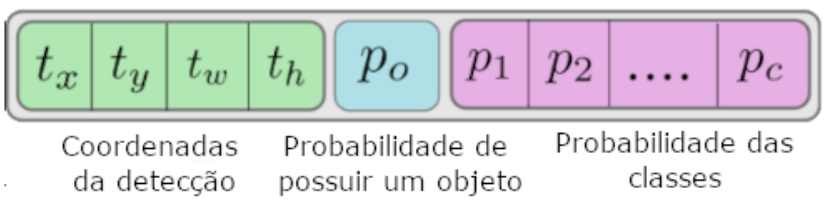

Como mencionado anteriormente, as coordenadas da região de detecção são relativas ao bloco do grid, logo as coordenadas absolutas podem ser obtidas conforme a Fig. 3. Onde $C_{x}$ e $C_{y}$ representam a coordenada absoluta do ponto superior esquerdo do bloco do grid. Os pontos $b_{x}, b_{y}, b_{w} \mathrm{e}$ $b_{h}$ representam a coordenada $x$ do centro, a coordenada $y$ do centro, a largura e altura absoluta da região de detecção, respectivamente. Os valores de $p_{w}$ e $p_{h}$ representam a largura e altura da ancora da região de detecção. E o $\sigma$ representa a função de ativação sigmoid.

A YOLOV4 Tiny é a versão comprimida da YOLOV4, possibilitando uma estrutura mais simples, para aplicações mobile e sistemas embarcados. A YOLOV4 Tiny tem como característica treinos e detecções mais rápidas devido a simplicidade da arquitetura. Ao contrário da YOLOV4, que possui 137 camadas convolucionais, a versão comprimida conta com 29 camadas convolucionais.

\section{Métricas para Avaliação de Desempenho}

Para avaliar o desempenho das técnicas utilizadas neste estudo, é necessário utilizar métricas de avaliação já estabelecidas na literatura. Esta seção apresenta as métricas utilizadas.
Fig. 3: Representação das coordenadas da região de detecção relativas e absolutas [6].

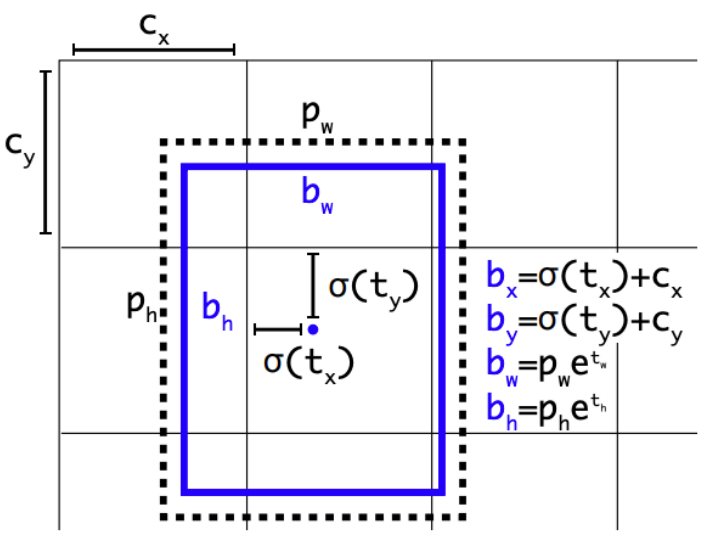

A Intersection Over Union ( $I o U)$, é uma métrica capaz de representar a similaridade entre a localização detectada e a localização real do objeto [18]. A Fig. 4 mostra que para obter o valor percentual de IoU, é necessário dividir a área de interseção pela área de união entre a região detectada e a região real do objeto.

Fig. 4: Cálculo da Intersection Over Union (IoU) [19].

$$
\text { IoU }=\frac{\text { Área da interseção }}{\text { Área da união }}
$$

Porém para análise de uma época, utiliza-se o Average $I o U$, que pode ser calculado conforme a Eq. (1).

$$
\text { Average } I o U=\frac{\sum I o U>T}{T P+F P}
$$

Onde $T P$ e $F P$ representam os verdadeiros positivos e os falsos positivos, respectivamente. Para que uma classificação seja considerada um verdadeiro positivo $(T P)$, a Intersection Over Union ( $I o U)$ deve ser maior que um limite pré-definido, $I o U>T$, onde $T$ é o limite. Neste estudo, utilizou-se o valor de 0,5 para $T$.

O recall é uma métrica utilizada para representar o percentual de detecções corretas e pode ser calculado conforme Eq. (2) [20].

$$
\text { recall }=\frac{T P}{T P+F N}
$$

A precision é uma métrica utilizada para avaliar a qualidade do modelo, para o cálculo pode ser visualizado na Eq. (3) [20]. 
Tabela III: Especificações de treinamento

$$
\text { precision }=\frac{T P}{T P+F P}
$$

A Average Precision ( $A P$ ) é uma métrica utilizada para expressar a precisão média para todos os valores de recall, ou seja, é a área sob a curva precision-recall da seguinte forma:

$$
A P=\int_{0}^{1} p(r) d r
$$

onde $p$ e $r$, representam a precision e recall respectivamente.

A última métrica utilizada neste artigo é o tempo de latência em milissegundos (ms), para a detecção do nadador. O tempo de latência, é tempo que o algoritmo leva para processar a imagem a partir do momento em que é requisitada, ou seja, quanto menor o tempo de latência mais rápido será a detecção dos objetos.

\section{EXPERIMENTOS}

Esta seção tem por objetivo mostrar o equipamento utilizado, a configuração dos parâmetros para cada algoritmo e os detalhes de treinamento.

\section{A. Treinamento dos Modelos}

A Tabela II apresenta as especificações técnicas do computador utilizado para treinamento dos dois algoritmos.

Tabela II: Especificações técnicas do computador utilizado

\begin{tabular}{cc}
\hline Sistema Operacional & Windows 10 Education 64 bits \\
Processador & i9-10900F @ $2.80 \mathrm{GHz}$ \\
Memória RAM & $128 \mathrm{~GB}$ DDR4 \\
Placa de Vídeo & RTX 2080 Ti $(11 \mathrm{~GB})$ \\
\hline
\end{tabular}

Para definir o número de épocas necessário para o treinamento do modelo, recomenda-se utilizar a multiplicação do número de classes por 2.000, porém, recomenda-se o número mínimo de 6.000 épocas [7]. Como o foco é a detecção de nadador, ou seja, apenas uma classe, a quantidade de filtros nas camadas convolucionais que precedem as camadas $Y O L O$ e o número de épocas devem ser ajustados. O número de filtros é calculado de acordo com a Eq. (5), logo utilizou-se 18 filtros e o mínimo de 6.000 épocas para treinamento.

$$
\text { filtros }=3 \times(\text { num.classes }+5)
$$

A Tabela III apresenta os hiper-parâmetros utilizados para o treinamento dos algoritmos, segundo os padrões apresentados em [7].

\begin{tabular}{ccc}
\hline Parâmetro & YOLO v4 & YOLO v4 Tiny \\
\hline Batch & 64 & 64 \\
Tamanho da imagem & $416 \times 416$ & $416 \times 416$ \\
Momentum & 0,949 & 0,9 \\
Decay & 0,0005 & 0,005 \\
Learning Rate & 0,001 & 0,00261 \\
Anchor Boxes & 9 & 9 \\
Épocas de treinamento & 6.000 & 6.000 \\
Tempo de treinamento & 4 horas & 1,2 horas \\
\hline
\end{tabular}

\section{Resultados}

Esta seção apresenta a discussão dos resultados obtidos, comparando os valores das métricas de avaliação para cada algoritmo.

Realizou-se a comparação dos resultados utilizando as métricas descritas na seção III-C, sendo elas a Average IoU, Recall, Average Precision (AP) e tempo de latência, utilizando o limite de IoU como $50 \%$.

A Fig. 5 apresenta os valores obtidos de Average IoU, indicando uma semelhança entre os resultados. Contudo a YOLO v4 atingiu seu valor máximo de $71,38 \%$ com 2.000 épocas, já a YOLO v4 Tiny atingiu seu valor máximo de $66,16 \%$ com 4.000 épocas. Portanto a YOLO v4 apresenta vantagem de $5,22 \%$ analisando os melhores resultados de ambos os algoritmos.

Fig. 5: Comparação da Average Intersection Over Union (IoU) entre as redes YOLO v4 e YOLO v4 Tiny

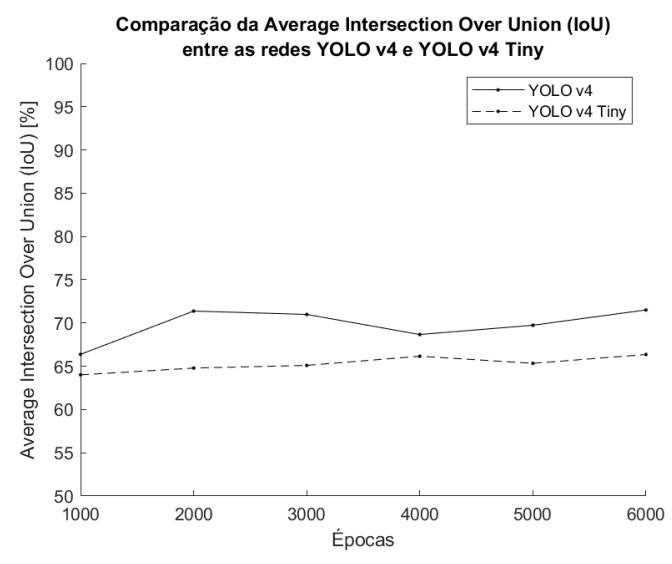

A Fig. 6 mostra a comparação dos valores de recall entre os algoritmos. Novamente, observa-se a semelhança entre os valores e os limites máximos de ambos os casos, estes acima de $90 \%$, sendo a YOLO v4 com o melhor resultado, atingindo $95 \%$.

A comparação da Average Precision é apresentada na Fig. 7, os dados apresentam valores próximos, e os limites 
Fig. 6: Comparação da recall entre as redes YOLO v4 e YOLO v4 Tiny

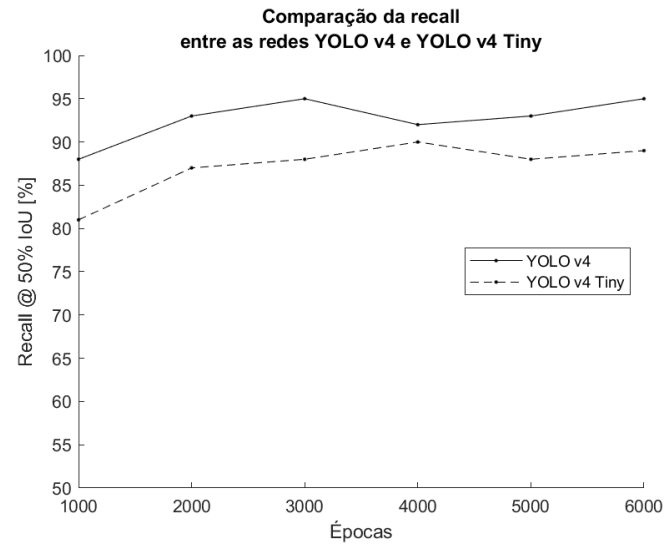

máximos acima de $90 \%$ em ambos os casos. Novamente, a YOLO v4 apresentou o melhor resultado, atingindo $94,28 \%$.

Fig. 7: Comparação da Average Precision (AP) entre as redes YOLO v4 e YOLO v4 Tiny

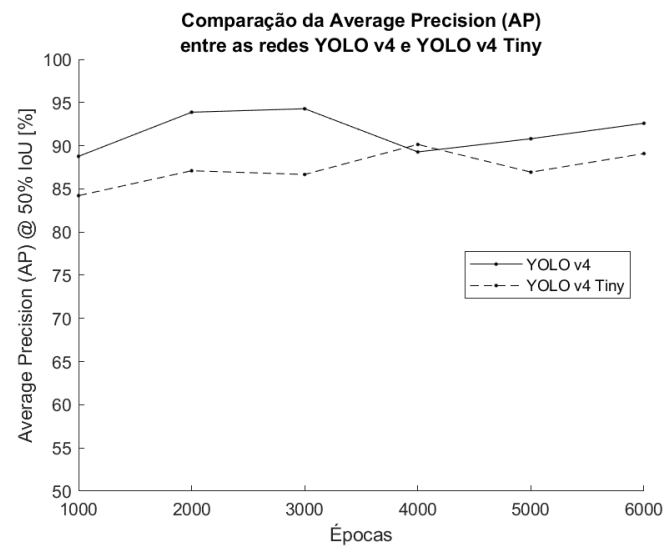

Já quando os resultados analisados levam em conta o tempo de latência (Fig. 8), observa-se a inversão do cenário, o tempo de latência da YOLO $v 4$ Tiny chega a ser 6,93 vezes menor que o tempo de latência da $Y O L O v 4$, mostrando vantagens em aplicações de tempo real.

Na Fig. 9, observa-se a detecção realizada pelos algoritmos. A YOLO v4 apresentou uma região bem definida e uma probabilidade de identificar um nadador superior ao da versão comprimida. Contudo, a YOLO v4 Tiny também apresentou uma região de detecção aceitável.

Também se realizou a análise para quando os nadadores estão parcialmente oclusos (Fig. 10). A versão comprimida conseguiu identificar apenas um dos nadadores oclusos, já a YOLO $v 4$ identificou ambos os nadadores parcialmente oclusos.
Fig. 8: Comparação do tempo de latência entre as redes $Y O L O$ $v 4$ e YOLO v4 Tiny
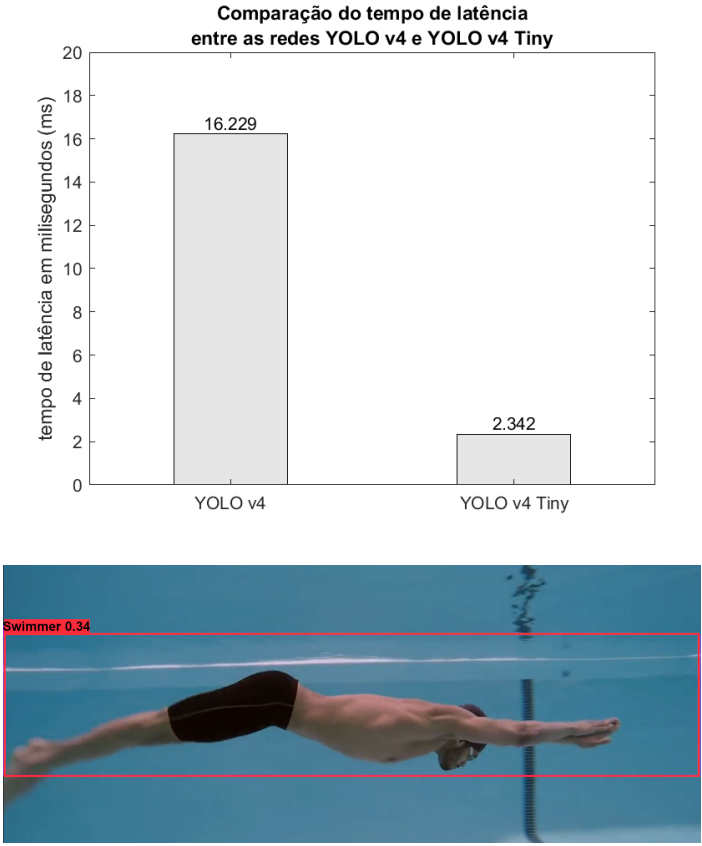

(a) Detecção YOLO v4 Tiny

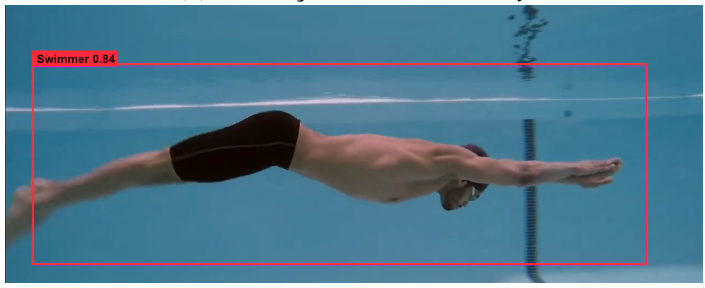

(b) Detecção $Y O L O$ v4

Fig. 9: Comparação da detecção realizadas pelos algoritmos

\section{CONCLUSÃO}

Este trabalho apresentou a coleta de dados de um dataset com imagens anotadas de nadadores em baixo d'água, treinamento e análise comparativa entre dois algoritmos de aprendizado profundo, YOLO v4 e YOLO v4 Tiny. Para comparação, utilizou-se as seguintes métricas de desempenho: Average IoU, Recall, Average Precision (AP) e tempo de latência, utilizando o limite de IoU como $50 \%$.

Ao analisar o desempenho das métricas, observa-se uma qualidade de detecção superior com a $Y O L O \nu 4$, o que era esperado por possuir uma estrutura mais complexa. Porém no tempo de latência, a versão comprimida usa a simplicidade de sua estrutura, para atingir valores até 6,93 vezes menor quando comparado com a $Y O L O v 4$, com o tempo de latência e tamanho reduzidos, a $Y O L O$ v4 Tiny se mostra apta para ser implementada em sistemas embarcados com foco em atividades de tempo real.

Observou-se falsos positivos para objetos como a divisória 


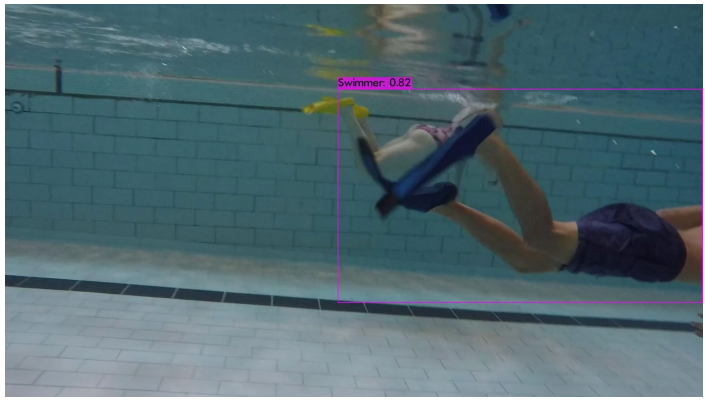

(a) Detecção YOLO v4 Tiny

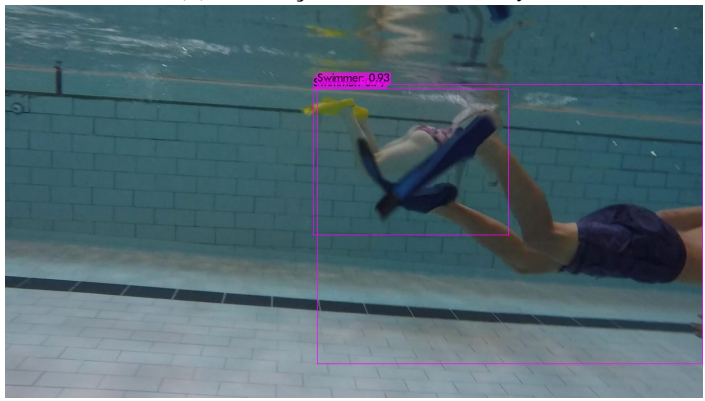

(b) Detecção $Y O L O v 4$

Fig. 10: Comparação da detecção de nadadores parcialmente oclusos realizadas pelos algoritmos

da raia e também dificuldade para detectar nadadores a partir de ângulos inferiores ou superiores, devido à falta de exemplares nas imagens da base de dados. Portanto enfatiza-se a importância dos trabalhos futuros, para expansão e melhoria das amostras da base de dados, tendo como objetivo aumentar a robustez e generalização do modelo.

Para os trabalhos futuros, propõe-se o aumento da base de dados utilizada para o treinamento, por meio da produção e anotação de mais vídeos de nadadores, buscando aumentar a robustez e generalização do modelo. Incorporação do algoritmo a um sistema embarcado, para que testes de qualidade e tempo de detecção possam ser conduzidos. E modelagem do controlador capaz de gerar referência de posição e velocidade para um veículo elétrico.

\section{AGRADECIMENTOS}

Os autores agradecem a Pontifícia Universidade Católica do Paraná (PUCPR) e a Fundação Araucária de apoio ao desenvolvimento científico e tecnológico do Paraná (PRONEX 042/2018) pelo financiamento desta pesquisa.

\section{REFERÊNCIAS}

[1] D. Benarab, T. Napoléon, A. Alfalou, A. Verney, and P. Hellard, "Swimmer's head detection based on a contrario and scaled composite jtc approaches," International Journal of Optics, vol. 2020, pp. 1-12, 042020.

[2] N. M. N. Adnan, M. N. A. A. Patar, H. Lee, S.-I. Yamamoto, L. Jong-Young, and J. Mahmud, "Biomechanical analysis using kinovea for sports application," IOP Conference Series: Materials Science and Engineering, vol. 342, p. 012097, apr 2018. [Online]. Available: https://doi.org/10.1088/1757-899x/342/1/012097
[3] M. Einfalt, D. Zecha, and R. Lienhart, "Activity-conditioned continuous human pose estimation for performance analysis of athletes using the example of swimming," in 2018 IEEE Winter Conference on Applications of Computer Vision (WACV), 2018, pp. 446-455.

[4] D. Benarab, T. Napoléon, A. Alfalou, A. Verney, and P. Hellard, "Optimized swimmer tracking system based on a novel multi-relatedtargets approach," Optics and Lasers in Engineering, vol. 89, pp. 195-202, 2017, 3DIM-DS 2015: Optical Image Processing in the context of 3D Imaging, Metrology, and Data Security. [Online]. Available: https://www.sciencedirect.com/science/article/pii/S0143816616300902

[5] K. de Langis, M. Fulton, and J. Sattar, "An analysis of deep object detectors for diver detection," 2020.

[6] J. Redmon and A. Farhadi, "Yolov3: An incremental improvement," arXiv, 2018

[7] A. Bochkovskiy, C. Wang, and H. M. Liao, "Yolov4: Optimal speed and accuracy of object detection," CoRR, vol. abs/2004.10934, 2020. [Online]. Available: https://arxiv.org/abs/2004.10934

[8] R. Huang, J. Pedoeem, and C. Chen, "Yolo-lite: A real-time object detection algorithm optimized for non-gpu computers," in 2018 IEEE International Conference on Big Data (Big Data), 2018, pp. 2503-2510.

[9] M. Everingham, L. Gool, C. K. Williams, J. Winn, and A. Zisserman, "The pascal visual object classes (voc) challenge," Int. J. Comput. Vision, vol. 88, no. 2, p. 303-338, Jun. 2010. [Online]. Available: https://doi.org/10.1007/s11263-009-0275-4

[10] T. Lin, M. Maire, S. J. Belongie, L. D. Bourdev, R. B. Girshick, J. Hays, P. Perona, D. Ramanan, P. Dollár, and C. L. Zitnick, "Microsoft COCO: common objects in context," CoRR, vol. abs/1405.0312, 2014. [Online]. Available: http://arxiv.org/abs/1405.0312

[11] M. J. Shafiee, B. Chywl, F. Li, and A. Wong, "Fast YOLO: A fast you only look once system for real-time embedded object detection in video," CoRR, vol. abs/1709.05943, 2017. [Online]. Available: http://arxiv.org/abs/1709.05943

[12] J. Redmon and A. Farhadi, "YOLO9000: better, faster, stronger," CoRR, vol. abs/1612.08242, 2016. [Online]. Available: http://arxiv.org/abs/1612.08242

[13] J. Redmon, S. Divvala, R. Girshick, and A. Farhadi, "You only look once: Unified, real-time object detection," 2016.

[14] C. Wang, H. M. Liao, I. Yeh, Y. Wu, P. Chen, and J. Hsieh, "Cspnet: A new backbone that can enhance learning capability of CNN," CoRR, vol. abs/1911.11929, 2019. [Online]. Available: http://arxiv.org/abs/1911.11929

[15] K. He, X. Zhang, S. Ren, and J. Sun, "Spatial pyramid pooling in deep convolutional networks for visual recognition," CoRR, vol. abs/1406.4729, 2014. [Online]. Available: http://arxiv.org/abs/1406.4729

[16] S. Liu, L. Qi, H. Qin, J. Shi, and J. Jia, "Path aggregation network for instance segmentation," CoRR, vol. abs/1803.01534, 2018. [Online]. Available: http://arxiv.org/abs/1803.01534

[17] A. Kathuria, "How to implement a yolo (v3) object detector from scratch in pytorch," https://blog.paperspace.com/how-to-implement-ayolo-object-detector-in-pytorch/, 2018, accessed: 2021-06-19.

[18] M. Rahman and Y. Wang, "Optimizing intersection-over-union in deep neural networks for image segmentation," vol. 10072, 12 2016, pp. 234 244.

[19] A. Rosebrock, "Intersection over union (iou) for object detection," https://www.pyimagesearch.com/2016/11/07/intersection-overunion-iou-for-object-detection/, 2016, accessed: 2021-06-18.

[20] B. S. dos Santos and M. T. A. Steiner, "Metodologia baseada em kdd para a classificação de mulheres fumantes quanto ao consumo de cigarros industrializados," Ph.D. dissertation, Pontifícia Universidade Católica do Paraná, Curitiba, 2020, accessed: 2021-06-16. 